\title{
Chemical Yields and Structural/Nucleosynthetic Evolution of Ultra Metal-poor Low-mass Stars
}

\author{
Simon W. Campbell*i \\ Monash Centre for Astrophysics, Buliding 28, Victoria 3800, Australia \\ E-mail: simon. campbell@monash.edu \\ John C. Lattanzio \\ Monash Centre for Astrophysics, Building 28, Victoria 3800, Australia \\ E-mail: john.lattanzio@monash.edu
}

\begin{abstract}
Until very recently all known stars with excessively low Fe abundances $([\mathrm{Fe} / \mathrm{H}] \lesssim-4)$ were observed to have very high $\mathrm{C}+\mathrm{N}+\mathrm{O}$ abundances $(\mathrm{eg}$. $[\mathrm{C} / \mathrm{Fe}]=4)$. Thus the total metallicities $(\mathrm{Z})$ were not actually very low. This suggested that low-mass stars could not form from material having extremely low Z. However Caffau et al. (2012) have just reported abundances of one Halo star that has extremely low $\mathrm{C}$ and $\mathrm{N}$ as well as $\mathrm{Fe}([\mathrm{Fe} / \mathrm{H}]=-5.0)$. Furthermore they predict that many more stars of this type should be found in the Halo. Thus, contrary to many theoretical predictions, it now appears that low-mass ultra Z-poor stars can indeed form. Motivated by this new information we report on our investigation into stellar evolution and nucleosynthesis of low-mass stars $\left(0.85<\mathrm{M}<3.0 \mathrm{M}_{\odot}\right)$ in the ultra low metallicity regime $([\mathrm{Fe} / \mathrm{H}] \leq-3.0)$, including models of stars with a pure Big Bang composition (i.e. $Z=0$ ). We have calculated the entire evolution of the $\mathrm{Z}=0$ and ultra metal-poor (UMP) models, from the ZAMS to the end of the TPAGB, including detailed nucleosynthesis. Some of the results from this study have been published previously. We find that many of the UMP and $\mathrm{Z}=0$ models experience violent evolutionary episodes not seen at higher metallicities. We refer to these events as 'Dual Flashes' and show that they can affect the surface composition and hence the chemical pattern in the yields. We provide tables of yields for our grid of models which are available in electronic format at CDS. Although subject to many uncertainties these are, as far as we are aware, the only yields available in this mass and metallicity range and should be of use to chemical evolution studies of the early Universe.
\end{abstract}

XII International Symposium on Nuclei in the Cosmos,

August 5-12, 2012

Cairns, Australia

\footnotetext{
*Speaker.

${ }^{\dagger}$ Yield tables are available at: http://cdsarc.u-strasbg.fr/viz-bin/qcat?J/A+A/490/769
} 


\section{Introduction}

The discovery of extremely metal-poor (EMP) stars in the Galactic Halo has naturally led to a renewed interest in the theoretical modelling of Population III and very low-metallicity stars. In particular the subset of these ancient stars that is observed to contain large amounts of carbon, the C-rich EMPs (CEMPs), which we define here as stars with $[\mathrm{C} / \mathrm{Fe}]>+0.7$, has attracted much stellar modelling work because their abundance patterns are difficult to explain with standard stellar evolution. These interesting objects also appear to comprise a large proportion of the EMPs $(\sim 10 \rightarrow 20 \%$; see eg. $[1,2,3]$ and the SAGA database $[4,5])$, suggesting that an additional (or modified) source of $\mathrm{C}$ production was active in the early Universe. The CEMPs also display variation in a range of other elements, such as s-process species [6]. A number of theories have been proposed to explain the various abundance patterns seen in CEMPs, ranging from pre-formation pollution via Pop III supernovae $[7,8]$ to self-pollution through peculiar evolutionary events $[9,10]$ to binary mass transfer $[11,12]$.

In the past the bulk of theoretical work had pointed to stellar initial mass functions (IMFs) peaked at very high masses $\left(>50 \mathrm{M}_{\odot}\right)$ in the early Universe $[13,14]$. This was due to the realisation that metal species important to cooling and fragmentation in star formation would not be available. However more recent work has been predicting ultra metal-poor (UMP) and primordial IMFs that include low-mass stars [15, 16]. Furthermore, the fact that ancient, low-mass halo stars having extremely low abundances of Fe have been directly observed suggests that the picture is not so simple. Cooling via small amounts of $\mathrm{C}$ and $\mathrm{O}$, which is very quickly available from Pop III supernovae, has now been shown to be enough to allow low-mass stars to form [17]. The study of [17] found a limit on the minimum amount of $\mathrm{C}$ needed to form low mass stars of $[\mathrm{C} / \mathrm{H}]=-2.9$. This fit well with the observations of the lowest metallicity EMP/UMP stars since all of those stars known at the time contained relatively high amounts of C. However the very recent discovery of a halo star having $[\mathrm{Fe} / \mathrm{H}]=-5.0$ but also $[\mathrm{C} / \mathrm{H}]=-3.8$ has cast doubt on this limit [18]. It now appears that low-mass stars were indeed able to form from ultra-Z-poor gas in the early Universe. Motivated by this new discovery we explore in this paper the nature of the chemical pollution produced by models of low- and intermediate-mass ( $\mathrm{LM}: \mathrm{M}<2.0 \mathrm{M}_{\odot}$ and $\mathrm{IM}: \mathrm{M} \geq 2.0 \mathrm{M}_{\odot}$ ) $\mathrm{UMP}$ and $\mathrm{Z}=0$ stars. We focus on stars having $[\mathrm{Fe} / \mathrm{H}] \leq-3.0$. As these stars don't produce elements heavier than Fe in situ (except possibly for s-process elements, see [20]), we assume that they have formed from gas clouds already enriched by Pop III supernovae that produced the near scaled-solar abundances of many elements seen in the 'normal' (non C-rich) UMP Halo stars. In terms of producing the excessive light element (ie. CNO) pollution evident in the CEMPs we keep an open mind as to whether they may have received their compositions through self-pollution events or through binary mass-transfer (be it from wind accretion or Roche lobe overflow). We note that some of the results of this work have been published in detail elsewhere as part of a series of papers $[19,20]$ and yield tables are available now online from CDS (catalogue J/A+A/490/769).

\section{The Grid of Models}

Our simulations were performed utilising two numerical codes - a stellar structure code and post-processing nucleosynthesis code. 
The stellar structure code used was the Monash version of the Monash/Mount Stromlo stellar evolution code (MONSTAR, see eg. [21, 22]). Convective mixing was followed with a timedependent (diffusive) mixing routine (similar to that described by [23]). This was necessary due to the violent evolutionary events (the H-flashes) that occur in models of $\mathrm{Z}=0$ and UMP stars, where the timescale for mixing becomes comparable to the evolutionary time steps, and thus the usual assumption of instantaneous mixing does not hold. Opacities from [24] were used for mid-range temperatures and those of [25] for low temperatures. Convective boundaries were always defined by the Schwarzschild criterion - no overshoot was applied. For mass loss the empirical formula of Reimers [26] was used during the RGB (with $\eta=0.4$ ). For the AGB we used the formula of Vassiliadis \& Wood [27]. As described below, all the models experience some self-pollution and always before or at the very beginning of the TPAGB phase. We find that the surfaces of the AGB models usually have metallicities approaching that of the LMC or even Solar (as defined by $Z=1-X-Y$ rather than $\mathrm{Fe}$ ). Since the stellar surfaces have (some of) the ingredients needed to form grains, we argue that using a standard mass loss formula is warranted, at least as a first approximation. We note that metallicity is also indirectly taken into account by the mass loss formulae, since they depend on bulk stellar properties (such as radius, luminosity, pulsation period), which vary significantly with metallicity.

The nucleosynthesis calculations were made with the Monash Stellar Nucleosynthesis code (MONSOON), a post-processing code which takes input from the MONSTAR code (eg. density, temperature, convective velocities). It solves a network of 506 nuclear reactions involving 74 nuclear species (see eg. [28, 29, 30]). Initial composition for the $\mathrm{Z}=0$ models was taken from the Standard Big Bang nucleosynthesis calculations of [31], whilst the initial composition for the UMP models was derived by mixing the ejecta from a $20 \mathrm{M}_{\odot} \mathrm{Z}=0$ supernova calculation (Limongi 2002, private communication) with varying amounts of Big Bang material from [31] to reach the desired $[\mathrm{Fe} / \mathrm{H}]$ values (for example $10^{6} \mathrm{M}_{\odot}$ of Big Bang material was required for $[\mathrm{Fe} / \mathrm{H}]=-4.0$ ).

Our grid of models covers the mass range: $\mathrm{M}=0.85,1.0,2.0,3.0 \mathrm{M}_{\odot}$ and the metallicity range: $[\mathrm{Fe} / \mathrm{H}]=-6.5,-5.45,-4.0,-3.0$, plus $\mathrm{Z}=0$. Figure 1 shows the grid of models.

\section{Results and Discussion}

We have calculated the entire evolution of the $\mathrm{Z}=0$ and UMP models, from the ZAMS to the end of the TPAGB, including extensive nucleosynthesis. We also calculated the integrated yields for each star. An example of one of our yield tables in shown in Table 1 and the full set is available online from CDS (catalogue $\mathrm{J} / \mathrm{A}+\mathrm{A} / 490 / 769$ ).

We found many of the models experienced violent nuclear burning episodes not seen at higher metallicities. We refer to these events as 'Dual Flashes' since they are characterised by nearly simultaneous peaks in both hydrogen and helium burning. These events have been reported by previous studies $[32,10]$ and have recently been explored with 3D hydrodynamical simulations $[33,34,35]$. The two types of dual flashes we define as the i) 'Dual Core Flash' (DCF, which occurs at the tip of the RGB), and ii) 'Dual Shell Flash' (DSF, which occurs during the first few pulses of the TPAGB phase). Some of the material processed by the Dual Flashes is dredged up causing significant surface pollution with a distinct chemical composition. This polluted material then contributes to the chemical patterns in the yields - especially in our lowest mass models $(0.85$ and 


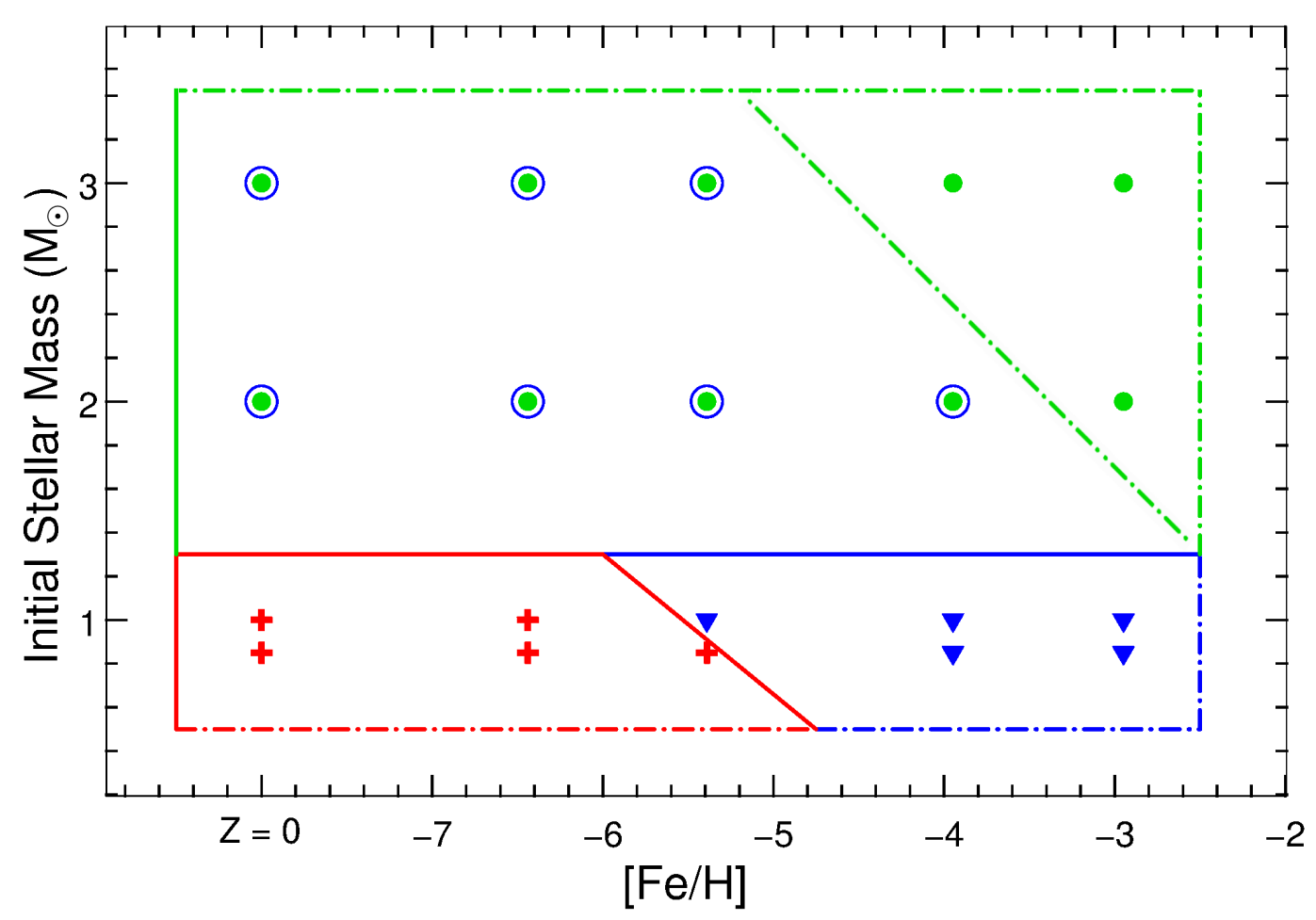

Figure 1: Mass-metallicity diagram showing our grid of models. Each symbol represents a single stellar model. The diagram also shows the dominant surface pollution channels we found in the models. Crosses (red) represent the dual core flash self-pollution Group 1, filled triangles (blue) the dual shell flash selfpollution Group 2 and filled circles (green) the AGB self-pollution Group 3. The open circles (blue) around the filled circles (green) indicate intermediate mass models that experienced dual shell flashes. Pollution from TDU (and HBB) easily dominates over the pollution from the DSF events at intermediate masses (M $\geq 2.0 \mathrm{M}_{\odot}$ ) so the yields of these models fall into the AGB group. The $\mathrm{Z}=0$ models are included at $[\mathrm{Fe} / \mathrm{H}]$ $=-8$.

$1.0 \mathrm{M}_{\odot}$ ). In our more massive models (2.0 and 3.0 $\mathrm{M}_{\odot}$ ) we find that third dredge-up (TDU) occurs. This repetitive pollution of the stellar envelope ends up dominating over the earlier DSF pollution. Moreover, CNO burning at the bottom of the convective envelope ('Hot Bottom Burning', HBB) is found to occur in these models. This cycles most of the dredged up CNO nuclei to ${ }^{14} \mathrm{~N}$, effectively erasing the chemical pattern of the DSF episodes. Thus these stars show chemical yield patterns reflecting the combination of TDU+HBB rather than that of the DSF episodes (see green dots surrounded by blue circles in Fig. 1). We summarise the self-pollution episodes over the whole grid of models by dividing them into three categories, defined by the evolutionary events/phases that dominate the chemical signature in the yields:

- Group 1 yields are dominated by the DCF events

- Group 2 are dominated by DSF events

- Group 3 are dominated by TDU+HBB 
Table 1: Part of the yield table for the $Z=0$ models. Yields are in mass-fraction, integrated over the whole wind mass-loss history of each star. Initial composition is included in the third column. The full set of yields is available online at CDS (catalogue $\mathrm{J} / \mathrm{A}+\mathrm{A} / 490 / 769)$.

\begin{tabular}{ccccccc}
\hline \hline Nuclide & $\mathrm{A}$ & Initial & $0.85 \mathrm{M}_{\odot}$ & $1.0 \mathrm{M}_{\odot}$ & $2.0 \mathrm{M}_{\odot}$ & $3.0 \mathrm{M} \odot$ \\
\hline${ }^{1} \mathrm{H}$ & 1 & $7.548 \mathrm{E}-01$ & $7.014 \mathrm{E}-01$ & $6.597 \mathrm{E}-01$ & $6.596 \mathrm{E}-01$ & $5.807 \mathrm{E}-01$ \\
${ }^{4} \mathrm{He}$ & 4 & $2.450 \mathrm{E}-01$ & $2.976 \mathrm{E}-01$ & $3.295 \mathrm{E}-01$ & $3.367 \mathrm{E}-01$ & $4.066 \mathrm{E}-01$ \\
${ }^{7} \mathrm{Li}$ & 7 & $3.130 \mathrm{E}-10$ & $4.704 \mathrm{E}-10$ & $1.263 \mathrm{E}-09$ & $4.491 \mathrm{E}-10$ & $4.887 \mathrm{E}-11$ \\
${ }^{12} \mathrm{C}$ & 12 & $0.000 \mathrm{E}+00$ & $2.598 \mathrm{E}-05$ & $1.844 \mathrm{E}-03$ & $1.309 \mathrm{E}-04$ & $4.882 \mathrm{E}-04$ \\
${ }^{13} \mathrm{C}$ & 13 & $0.000 \mathrm{E}+00$ & $7.778 \mathrm{E}-06$ & $3.619 \mathrm{E}-04$ & $3.034 \mathrm{E}-05$ & $1.152 \mathrm{E}-04$ \\
${ }^{14} \mathrm{~N}$ & 14 & $0.000 \mathrm{E}+00$ & $2.437 \mathrm{E}-04$ & $3.919 \mathrm{E}-03$ & $3.432 \mathrm{E}-03$ & $1.166 \mathrm{E}-02$ \\
${ }^{16} \mathrm{O}$ & 16 & $0.000 \mathrm{E}+00$ & $5.034 \mathrm{E}-04$ & $4.333 \mathrm{E}-03$ & $4.885 \mathrm{E}-05$ & $1.516 \mathrm{E}-04$ \\
${ }^{19} \mathrm{~F}$ & 19 & $0.000 \mathrm{E}+00$ & $1.848 \mathrm{E}-09$ & $6.225 \mathrm{E}-06$ & $2.879 \mathrm{E}-10$ & $1.188 \mathrm{E}-09$ \\
${ }^{20} \mathrm{Ne}$ & 20 & $0.000 \mathrm{E}+00$ & $2.485 \mathrm{E}-07$ & $1.726 \mathrm{E}-06$ & $2.737 \mathrm{E}-05$ & $1.386 \mathrm{E}-04$ \\
${ }^{23} \mathrm{Na}$ & 23 & $0.000 \mathrm{E}+00$ & $1.291 \mathrm{E}-09$ & $1.131 \mathrm{E}-05$ & $1.294 \mathrm{E}-05$ & $9.539 \mathrm{E}-05$ \\
${ }^{24} \mathrm{Mg}$ & 24 & $0.000 \mathrm{E}+00$ & $3.838 \mathrm{E}-11$ & $1.362 \mathrm{E}-06$ & $1.865 \mathrm{E}-07$ & $2.630 \mathrm{E}-07$ \\
${ }^{25} \mathrm{Mg}$ & 25 & $0.000 \mathrm{E}+00$ & $1.459 \mathrm{E}-08$ & $3.166 \mathrm{E}-07$ & $1.756 \mathrm{E}-06$ & $1.562 \mathrm{E}-05$ \\
${ }^{26} \mathrm{Mg}$ & 26 & $0.000 \mathrm{E}+00$ & $2.475 \mathrm{E}-08$ & $4.065 \mathrm{E}-08$ & $8.159 \mathrm{E}-06$ & $6.889 \mathrm{E}-05$ \\
${ }^{26} \mathrm{Al}$ & 26 & $0.000 \mathrm{E}+00$ & $3.182 \mathrm{E}-11$ & $3.364 \mathrm{E}-10$ & $3.085 \mathrm{E}-07$ & $1.487 \mathrm{E}-06$ \\
\hline
\end{tabular}

In Figure 1 we show the mass and metallicity ranges in which each type of pollution dominates.

We have compared our $\mathrm{C}$ yields with observations of CEMP stars. At the lowest metallicities ( $[\mathrm{Fe} / \mathrm{H}] \lesssim-4.0$ ) we find the yields to contain $\sim 1$ to 2 dex too much carbon, in agreement with all previous studies (eg. $[9,36,37])$. At higher metallicities $([\mathrm{Fe} / \mathrm{H}] \sim-3.0)$, where the observed data set is much larger, all our models produce yields with $[\mathrm{C} / \mathrm{Fe}]$ values consistent with those observed in the most C-rich CEMPs. However it is only the low-mass models that undergo the Dual Shell Flash (which occurs at the start of the TPAGB) that can best reproduce the $\mathrm{C}$ and $\mathrm{N}$ observations simultaneously. Normal Third Dredge-Up (TDU) cannot reproduce the observations because at these metallicities intermediate mass models $\left(\mathrm{M} \gtrsim 2 \mathrm{M}_{\odot}\right)$ suffer $\mathrm{HBB}$ which converts the $\mathrm{C}$ to $\mathrm{N}$ thus lowering $[\mathrm{C} / \mathrm{N}]$ well below the observations, whilst if TDU were to occur in the low-mass ( $\mathrm{M} \leq 1 \mathrm{M}_{\odot}$ ) models (we do not find it to occur in our models), the yields would be expected to be C-rich only, which is at odds with the 'dual pollution' of $\mathrm{C}$ and $\mathrm{N}$ generally observed in the CEMPs (we note that the SAGA database has a compilation of halo star abundances [5]).

We also find that the proportion of CEMP stars should continue to increase at lower metallicities, based on the results that some of the low mass UMP models already have polluted surfaces by the core helium burning phase, and that there are more C-producing evolutionary episodes at these metallicities. This is indeed observed [5], although the current sample in the UMP regime is small.

With regards to the Caffau star (SDSS J102915+172927, [18]), which has a mass $\leq 0.7 \mathrm{M}_{\odot}$, we would not predict that it has had any form of surface self-pollution since it is currently in the MS or subgiant branch phase [18], well before a dual core flash would occur.

Finally we note that, although subject to many uncertainties, the $\mathrm{Z}=0$ and UMP yields presented here are the only yields currently available in the low and intermediate mass range. They are important for chemical evolution simulations that include the early Universe (eg. [38]). 


\section{References}

[1] Beers, T. \& Christlieb, N. 2005, The Discovery and Analysis of Very Metal-Poor Stars in the Galaxy, ARA\&A, 43, 531

[2] Cohen, J. G., Shectman, S., Thompson, I., McWilliam, A., Christlieb, N., Melendez, J., Zickgraf, F.J. , Ramírez, S., Swenson, A., 2005, The Frequency of Carbon Stars among Extremely Metal-poor Stars, ApJL, 633, L109

[3] Lucatello, S., Beers, T. C., Christlieb, N., Barklem, P. S., Rossi, S., Marsteller, B., Sivarani, T., Lee, Y. S., 2006, The Frequency of Carbon-enhanced Metal-poor Stars in the Galaxy from the HERES Sample, ApJL, 652, L37

[4] Suda, T., Katsuta, Y., Yamada, S., Suwa, T., Ishizuka, C., Komiya, Y., Sorai, K., Aikawa, M. and Fujimoto, M. Y, 2008, Stellar Abundances for the Galactic Archeology (SAGA) Database Compilation of the Characteristics of Known Extremely Metal-Poor Stars, PASJ, 60, 1159

[5] Suda, T., Yamada, S., Katsuta, Y., Komiya, Y., Ishizuka, C., Aoki, W. and Fujimoto, M. Y., 2011, The Stellar Abundances for Galactic Archaeology (SAGA) data base - II. Implications for mixing and nucleosynthesis in extremely metal-poor stars and chemical enrichment of the Galaxy, MNRAS, 412, 843

[6] Aoki, W., Bisterzo, S., Gallino, R., Beers, T. C. , Norris, J. E., Ryan, S. G., Tsangarides, S., 2006, Carbon-enhanced Metal-poor Stars: Osmium and Iridium Abundances in the Neutron-Capture-enhanced Subgiants CS 31062-050 and LP 625-44, ApJL, 650, L127

[7] Limongi, M., Chieffi, A. and Bonifacio, P., 2003, On the Origin of HE 0107-5240, the Most Iron-deficient Star Presently Known, ApJL, 594, L123

[8] Umeda, H. and Nomoto, K., 2005, Variations in the Abundance Pattern of Extremely Metal-Poor Stars and Nucleosynthesis in Population III Supernovae, ApJ, 619, 427

[9] Fujimoto, M. Y., Ikeda, Y. and Iben, I. J., 2003, The Origin of Extremely Metal-poor Carbon Stars and the Search for Population III, ApJ, 529, L25

[10] Weiss, A., Schlattl, H., Salaris, M. and Cassisi, S. , 2004, Models for extremely metal-poor halo stars, A\&A, 422, 217

[11] Suda, T., Aikawa, M., Machida, M. N., Fujimoto, M. Y. and Iben, I. J., 2004, Is HE 0107-5240 A Primordial Star? The Characteristics of Extremely Metal-Poor Carbon-Rich Stars, ApJ, 611, 476

[12] Komiya, Y., Suda, T., Minaguchi, H., Shigeyama, T., Aoki, W. and Fujimoto, M. Y., 2007, The Origin of Carbon Enhancement and the Initial Mass Function of Extremely Metal-poor Stars in the Galactic Halo, ApJ, 658, 367

[13] Abel, T., Anninos, P., Norman, M. L., Zhang, Y., 1998, First Structure Formation. I. Primordial Star-forming Regions in Hierarchical Models, ApJ, 508, 518

[14] Bromm, V., Coppi, P. S., Larson, R. B., 1999, Forming the First Stars in the Universe: The Fragmentation of Primordial Gas, ApJL, 527, L5

[15] Nakamura, F. and Umemura, M., 2001, On the Initial Mass Function of Population III Stars, ApJ, 548,19

[16] Greif, T. H., Springel, V., White, S. D. M., Glover, S. C. O., Clark, P. C., Smith, R. J., Klessen, R. S., Bromm, V., 2011, Simulations on a Moving Mesh: The Clustered Formation of Population III Protostars, ApJ, 737, 75 
[17] Bromm, V. and Loeb, A., 2003, The formation of the first low-mass stars from gas with low carbon and oxygen abundances, NATURE, 425, 812

[18] Caffau, E., Bonifacio, P., François, P., Spite, M., Spite, F., Zaggia, S., Ludwig, H.-G., Steffen, M., Mashonkina, L., Monaco, L., Sbordone, L., Molaro, P., Cayrel, R., Plez, B., Hill, V., Hammer, F., Randich, S., 2012, A primordial star in the heart of the Lion, A\&A, 542, A51

[19] Campbell, S. W. and Lattanzio, J. C., 2008, Evolution and nucleosynthesis of extremely metal-poor and metal-free low-and intermediate-mass stars. I. Stellar yield tables and the CEMPs, A\&A, 490, 769

[20] Campbell, S. W., Lugaro, M. and Karakas, A. I., Evolution and nucleosynthesis of extremely metal-poor and metal-free low- and intermediate-mass stars. II. s-process nucleosynthesis during the core He flash, A\&A, 522, L6

[21] Wood, P. R. and Zarro, D. M., 1981, Helium-shell flashing in low-mass stars and period changes in mira variables, ApJ, 247, 247

[22] Frost, C. A. and Lattanzio, J. C., 1996, On the Numerical Treatment and Dependence of the Third Dredge-up Phenomenon, ApJ, 473, 383

[23] Meynet, G., Maeder, A., Mowlavi, N., 2004, Diffusion in stellar interiors: Critical tests of three numerical methods, $A \& A, 416,1023$

[24] Iglesias, C. A. and Rogers, F. J., 1996, Updated Opal Opacities, ApJ, 464, 943

[25] Ferguson, J. W., Alexander, D. R., Allard, F., Barman, T., Bodnarik, J. G., Hauschildt, P. H., Heffner-Wong, A., Tamanai, A., 2005, Low-Temperature Opacities, ApJ, 623, 585

[26] Reimers, D., 1975, Circumstellar absorption lines and mass loss from red giants, Memoires of the Societe Royale des Sciences de Liege, 8, 369

[27] Vassiliadis, E. and Wood, P. R., Evolution of low-and intermediate-mass stars to the end of the asymptotic giant branch with mass loss, ApJ, 413, 641

[28] Cannon, R. C., 1993, Massive Thorne-Zytkow Objects - Structure and Nucleosynthesis, MNRAS, 263, 817

[29] Lattanzio, J., Frost, C., Cannon, R., Wood, P. R., 1996, Hot bottom burning in intermediate mass stars, MmSAI, 67, 729

[30] Lugaro, M., Ugalde, C., Karakas, A. I., Görres, J., Wiescher, M., Lattanzio, J. C., Cannon, R. C., 2004, Reaction Rate Uncertainties and the Production of ${ }^{19} \mathrm{~F}$ in Asymptotic Giant Branch Stars, ApJ, 615,934

[31] Coc, A., Vangioni-Flam, E., Descouvemont, P., Adahchour, A., Angulo, C., 2004, Updated Big Bang Nucleosynthesis Compared with Wilkinson Microwave Anisotropy Probe Observations and the Abundance of Light Elements, ApJ, 600, 544

[32] Fujimoto, M. Y., Iben, I. J., Hollowell, D., 1990, Helium flashes and hydrogen mixing in low-mass population III stars, ApJ, 349, 580

[33] Mocák, M., Campbell, S. W., Müller, E., Kifonidis, K., 2010, The core helium flash revisited. III. From Population I to Population III stars, A\&A, 520, A114

[34] Stancliffe, R. J., Dearborn, D. S. P., Lattanzio, J. C., Heap, S. A., Campbell, S. W., 2011, Three-dimensional Hydrodynamical Simulations of a Proton Ingestion Episode in a Low-metallicity Asymptotic Giant Branch Star, ApJ, 742, 121 
[35] Herwig, F., Pignatari, M., Woodward, P. R., Porter, D. H., Rockefeller, G., Fryer, C. L., Bennett, M., Hirschi, R., 2011, Convective-reactive Proton- ${ }^{12}$ C Combustion in Sakurai's Object (V4334 Sagittarii) and Implications for the Evolution and Yields from the First Generations of Stars, ApJ, 727, 89

[36] Schlattl, H., Cassisi, S., Salaris, M., Weiss, A., 2001, On the Helium Flash in Low-Mass Population III Red Giant Stars, ApJ, 559, 1082

[37] Chieffi, A., Domínguez, I., Limongi, M., Straniero, O., 2001, Evolution and Nucleosynthesis of Zero-Metal Intermediate-Mass Stars, ApJ, 554, 1159

[38] Kobayashi, C., Karakas, A. I., Umeda, H., 2011, The evolution of isotope ratios in the Milky Way Galaxy, MNRAS, 414, 3231 